\title{
Demixing in mesoscopic boson-fermion clouds inside cylindrical harmonic traps: quantum phase diagram and role of temperature
}

\author{
Z. Akdeniz ${ }^{1,2}$, A. Minguzzi ${ }^{1}$, P. Vignolo ${ }^{1}$, and M. P. Tosi ${ }^{1}$ \\ (1) NEST-INFM and Scuola Normale Superiore, Piazza dei Cavalieri 7, I-56126 Pisa, Italy \\ (2) Department of Physics, University of Istanbul, Istanbul, Turkey
}

\begin{abstract}
We use a semiclassical three-fluid thermodynamic model to evaluate the phenomena of spatial demixing in mesoscopic clouds of fermionic and bosonic atoms at high dilution under harmonic confinement, assuming repulsive boson-boson and boson-fermion interactions and including account of a bosonic thermal cloud at finite temperature $T$. The finite system size allows three different regimes for the equilibrium density profiles at $T=0$ : a fully mixed state, a partially mixed state in which the overlap between the boson and fermion clouds is decreasing, and a fully demixed state where the two clouds have zero overlap. We propose simple analytical rules for the two cross-overs between the three regimes as functions of the physical system parameters and support these rules by extensive numerical calculations. A universal "phase diagram" expressed in terms of simple scaling parameters is shown to be valid for the transition to the regime of full demixing, inside which we identify several exotic configurations for the two phase-separated clouds in addition to simple ones consisting of a core of bosons enveloped by fermions and vice versa. With increasing temperature the main role of the growing thermal cloud of bosons is to transform some exotic configurations into more symmetric ones, until demixing is ultimately lost. For very high values of boson-fermion repulsive coupling we also report demixing between the fermions and the thermally excited bosons.
\end{abstract}

PACS numbers: 05.30.-d, 03.75.Fi, 73.43.Nq, 67.40.Kh

\section{INTRODUCTION}

After the realization of Bose-Einstein condensation (BEC) in atomic gases [1], one of the most challenging endeavours in experiments on cold atoms is the cooling of fermionic isotopes of alkali atoms down to expected the superfluid transition. Theoretical estimates based on the BCS model 22 indicate that the temperature for the superfluid transition should be much lower than the Fermi temperature $T_{f}$, which is of the order of the BEC transition temperature. In fact, cooling fermions is harder than cooling bosons: the main difficulty arises from Fermi statistics, as $s$-wave collisions between spin-polarized fermions in a magnetic trap are forbidden by the Pauli principle. A common strategy uses sympathetic cooling, which is based on $s$-wave collisions between the fermions and a second gaseous component made either of fermions in a different internal state or of bosons. The latter choice seems to minimize the effects of Pauli blocking, which limit the process of cooling two fermionic components [3]. Several experiments are currently in progress on trapping and cooling various boson-fermion mixtures, i.e. ${ }^{6} \mathrm{Li}^{7} \mathrm{Li}$ [4, 5], ${ }^{6} \mathrm{Li}^{23} \mathrm{Na}$ [6] and ${ }^{40} \mathrm{~K}-{ }^{87} \mathrm{Rb}[8]$. The lowest temperature attained in these experiments so far is about $0.2 T_{f}$.

From the theoretical point of view a mixture of condensed bosons and fermions in the normal state is already an interesting system to study, because it can show spatial demixing of the two components [8]. In the homogeneous gas at $T=0$ this is an example of a quantum phase transition, that is a phase transition induced by the interactions [9]: on increasing the boson-fermion repulsion the system minimizes its total energy by placing the bosons and the fermions (or boson-fermion mixtures of different compositions) in different regions of space [10], even though this implies a high cost of kinetic energy at the interface. Boson-fermion demixing in a quasi-spherical trap has been studied by Nygaard and Mølmer [11], while the conditions for demixing inside a spherical trap have been set out by two of us [12] for $N_{b}=N_{f}$ and by Miyakawa et al. [13] for $N_{f} \ll N_{b}$, with $N_{b}$ and $N_{f}$ the numbers of bosons and fermions in the trap. The conditions for observing demixing in the Paris experiment on a ${ }^{6} \mathrm{Li}-{ }^{7} \mathrm{Li}$ mixture in a cigar-shaped trap [5] has been analyzed by Akdeniz et al. [14]. There are, of course, important consequences of finite system size on demixing in trapped mixtures, relative to the case of a homogeneous one. In particular, the transition is spread out in a finite system and the anisotropy of the trap acts differently on the two types of atoms (expressions such as phase transition and phase diagram will nevertheless continue to be used for brevity in the following). Locating the onset of partial demixing is also relevant to the practicalities of fermion cooling, since at that point the diminishing overlap between the two clouds will start reducing the effectiveness of the collisional transfer.

In this paper we give the general conditions under which phase separation of bosons and fermions occurs inside a harmonic trap at varying anisotropy and relative numbers of the two components. Within a mean-field model we make an extensive study of the equilibrium density profiles of the bosonic and fermionic component, ranging from the fully mixed state at small values of the scattering lengths to the partially demixed state at intermediate values of the 
coupling constants and finally to the regime of full phase separation. We show that the results can be understood on the basis of scaling laws and simply summarized into a phase diagram, which is expressed in terms of scaling parameters of the system at zero temperature. In the phase-separated regime we find several configurations having different symmetry and topology, some of which are metastable, and investigate the role of the anisotropy of the confinement in determining the minimum-energy configurations. We then extend our analysis to finite temperature, finding that on increasing temperature some phase-separated configurations turn or decay into others of higher symmetry before phase separation disappears. Finally, we show that phase separation between the fermions and a bosonic thermal cloud is also possible in principle on further increase of the boson-fermion scattering length.

The paper is organized as follows. In Sec. II we describe the model that we have used and give the limits of its applicability, together with a schematic description of the numerical method employed to find the phase-separated configurations. Sec. III summarizes the conditions under which phase separation occurs at $T=0$, illustrates various configurations which can be found in the phase-separated regime and gives the phase diagram for the lowest energy configurations. Sec. IV illustrates the effect of temperature on the configurations obtained for both small and large boson-fermion scattering length. Finally, Sec. $\mathrm{V}$ gives a summary and some concluding remarks.

\section{THE METHOD}

We describe the boson-fermion mixture by means of the particle density profiles, which are $n_{c}(\mathbf{r})$ for the condensate, $n_{n c}(\mathbf{r})$ for the bosonic thermal cloud and $n_{f}(\mathbf{r})$ for the fermions. The components are subject to axially symmetric confining potentials given by

$$
V_{b, f}^{e x t}(\mathbf{r})=m_{b, f} \omega_{b, f}^{2}\left(r_{\perp}^{2}+\lambda_{b, f}^{2} z^{2}\right) / 2
$$

where $m_{b, f}$ are the atomic masses, $\omega_{b, f}$ the trap frequencies and $\lambda_{b, f}$ the trap anisotropies. We evaluate the density profiles in a mean-field model using the Thomas-Fermi approximation for the condensate and the Hartree-Fock approximation for the other clouds [15, 16].

The Thomas-Fermi approximation assumes that the number of condensed bosons is large enough that the kinetic energy term in the Gross-Pitaevskii equation may be neglected [17. It yields

$$
n_{c}(\mathbf{r})=\left[\mu_{b}-V_{b}^{e x t}(\mathbf{r})-2 g n_{n c}(\mathbf{r})-f n_{f}(\mathbf{r})\right] / g
$$

for positive values of the function in the square brackets and zero otherwise. Here, $\mu_{b}$ is the chemical potential of the bosons and the coupling constants are $g=4 \pi \hbar^{2} a_{b b} / m_{b}$ and $f=2 \pi \hbar^{2} a_{b f} / m_{r}$, with $a_{b b}$ and $a_{b f}$ the boson-boson and boson-fermion scattering lengths and $m_{r}=m_{b} m_{f} /\left(m_{b}+m_{f}\right)$ the reduced mass. The Hartree-Fock approximation, on the other hand, treats the thermal boson cloud and the fermion cloud as ideal gases subject to effective potentials $V_{b, f}^{e f f}$, that is

$$
n_{n c, f}(\mathbf{r})=\int \frac{d^{3} p}{(2 \pi \hbar)^{3}}\left\{\exp \left[\left(\frac{p^{2}}{2 m_{b, f}}+V_{b, f}^{e f f}(\mathbf{r})-\mu_{b, f}\right) / k_{B} T\right] \mp 1\right\}^{-1}
$$

where

$$
V_{b}^{e f f}(\mathbf{r})=V_{b}^{e x t}(\mathbf{r})+2 g n_{c}(\mathbf{r})+2 g n_{n c}(\mathbf{r})+f n_{f}(\mathbf{r})
$$

and

$$
V_{f}^{e f f}(\mathbf{r})=V_{f}^{e x t}(\mathbf{r})+f n_{c}(\mathbf{r})+f n_{n c}(\mathbf{r}) .
$$

The chemical potentials $\mu_{b, f}$ are determined by requiring that the volume integrals of the densities $n_{c}(\mathbf{r})+n_{n c}(\mathbf{r})$ and $n_{f}(\mathbf{r})$ should be equal to the numbers $N_{b}$ and $N_{f}$ of particles.

The model is valid for the bosons when the diluteness condition $n_{c} a_{b b}^{3} \ll 1$ holds and the temperature of the mixture is sufficiently below the condensation temperature. The fermionic component has been taken as dilute spinpolarized gas, for which the fermion-fermion $s$-wave scattering processes are inhibited by the Pauli principle and $p$-wave scattering is negligible [18]. The condition $k_{f} a_{b f} \ll 1$ with $k_{f}$ the Fermi wave number should hold in the mixed regime, but this is not a constraint in the regime of phase separation where the boson-fermion overlap is rapidly dropping.

The density profiles at fixed numbers $N_{b}$ and $N_{f}$ are determined numerically by a self-consistent solution of the above equations. The profiles are obtained in two self-consistency loops: first, Eqs. (2) and (3) are solved at fixed chemical 
potentials starting from some initial guesses for the densities; then the chemical potentials are found iteratively by a standard algorithm for multi-variable minimization. Whereas in the mixed state the density profiles at convergence do not depend on the initial choice, different configurations are found in the phase-separation regime for the same values of the parameters by varying the initial conditions. We have used this fact to search for several possible metastable configurations, which will be illustrated in Sec. III B below.

\section{PHASE DIAGRAM AT ZERO TEMPERATURE}

As is evident from the preceding section, a boson-fermion mixture under confinement is characterized by a large number of parameters. The aim of this section is to analyze what is the effect of varying them independently and to provide a unified understanding of the results using scaling laws.

The interaction energy grows on increasing the values of the boson-boson and boson-fermion coupling constants. There exists a threshold beyond which the total energy is minimized by a configuration in which the two components are spatially separated. The transition to the phase-separated regime is smooth owing to the finite size of the confined gas, and three different states are indeed recognizable: mixed, partially demixed, and fully demixed. Depending on the value of the relative strength of the two coupling constants, it may happen that either the fermions or the bosons are pushed away from the center of the trap.

\section{A. Partial demixing}

The boson-fermion interaction energy is proportional to the overlap between bosonic and fermionic clouds and at $T=0$ we have

$$
E_{\text {int }}=f \int d^{3} r n_{c}(\mathbf{r}) n_{f}(\mathbf{r})
$$

We locate the onset of partial demixing by looking at the point where the boson-fermion overlap starts to decrease with increasing $a_{b f} / a_{b b}$.

In the following we focus on the simplest case where only one scattering length is varied, which also seems realistic from an experimental point of view. Then the most efficient way to approach phase separation is by changing $a_{b f}$ (see Sec. III B below). The behavior of the interaction energy as a function of the ratio $a_{b f} / a_{b b}$ at fixed $a_{b b}$ is shown in Fig. 1(a).

The position of the maximum of the interaction energy at fixed $a_{b b}$ can be estimated analytically from the condition $\partial E_{\text {int }} / \partial f=0$, by using the approximate expressions $n_{b, f} \approx N_{b, f} /\left(4 \pi R_{b, f}^{3} / 3\right)$ and the values for the cloud radii $R_{f}$ and $R_{b}$ as obtained in the absence of boson-fermion interactions, $R_{f}=\left(48 N_{f} / \lambda_{f}\right)^{1 / 6} a_{f}$ and $R_{b}=\left(15 \lambda_{b} N_{b} a_{b b} / a_{b}\right)^{1 / 5} a_{b} / \lambda_{b}^{1 / 3}$ with $a_{b, f}=\left(\hbar / m_{b, f} \omega_{b, f}\right)^{1 / 2}$. The expression for the position of the maximum is

$$
\left.\frac{a_{b f}}{a_{b b}}\right|_{\max }=\left(c_{1} \frac{N_{f}^{1 / 2}}{N_{b}^{2 / 5}}+c_{2} \frac{N_{b}^{2 / 5}}{N_{f}^{1 / 3}}\right)^{-1}
$$

where

$$
c_{1}=\frac{15^{3 / 5}}{48^{1 / 2}} \frac{\lambda_{f}^{1 / 2}}{\lambda_{b}^{2 / 5}} \frac{m_{f}^{3 / 2} \omega_{f}}{2 m_{r} m_{b}^{1 / 2} \omega_{b}}\left(\frac{a_{b b}}{a_{b}}\right)^{3 / 5}
$$

and

$$
c_{2}=\frac{48^{1 / 3}}{15^{3 / 5}}\left(\frac{6}{\pi}\right)^{2 / 3} \frac{\lambda_{b}^{2 / 5}}{\lambda_{f}^{1 / 3}} \frac{m_{b} \omega_{b}}{2 m_{r} \omega_{f}}\left(\frac{a_{b b}}{a_{b}}\right)^{2 / 5} .
$$

We recognise in Eq. (7) a geometric combination of two scaling parameters: $c_{1} N_{f}^{1 / 2} / N_{b}^{2 / 5}$ is dominant in the case $N_{b} \ll N_{f}$ while $c_{2} N_{b}^{2 / 5} / N_{f}^{1 / 3}$ was previously identified [19] in the regime $N_{f} \ll N_{b}$. The predictions obtained from Eq. (7) are indicated in Fig. 1(a) by vertical arrows. There clearly is very good agreement between the analytical estimate and the numerical results. 


\section{B. Full demixing}

The regime of full demixing is taken to be reached when the boson-fermion overlap becomes negligible. As is shown in Fig. 1(b), within the three-fluid model the transition point does not depend on the number of bosons in the trap. This can also be predicted from the results obtained by Viverit et al. [10] for the homogeneous mixture, by using the values of the densities taken at the center of the trap. Within the same approximation for the fermion density as in Sec. III A we obtain the condition for phase separation at $T=0$ as

$$
\alpha k_{f} a_{b b}>\left(\frac{a_{b b}}{a_{b f}}\right)^{2}
$$

where $k_{f}=\left(48 \lambda_{f} N_{f}\right)^{1 / 6} / a_{f}$ and $\alpha=\left[3^{1 / 3} /(2 \pi)^{2 / 3}\right] m_{b} m_{f} /\left(4 m_{r}^{2}\right)$. Equation (10) is valid for different numbers of bosons and fermions as well as for different atomic masses and trap frequencies, and agrees very well with the phaseseparation criterion given earlier by two of us 12 for the case where $m_{f}=m_{b}$ and $\omega_{f}=\omega_{b}$. It confirms that the two relevant parameters for describing the transition to the fully demixed regime are $a_{b f} / a_{b b}$ and $\alpha k_{f} a_{b b}$. We also immediately find that the value of $a_{b f}$ at the transition point scales as $N_{f}^{-1 / 12}$ at fixed $a_{b b}$ : this result is illustrated in the inset in Fig. 1(a).

Equation (10) has been verified by the numerical solution of the three-fluid model for a variety of different sets of parameters. First of all we have studied the location of phase separation in parameter space by varying the scattering lengths over much wider ranges than allowed by the usual off-resonant values (e.g. in the ${ }^{6} \mathrm{Li}-{ }^{7} \mathrm{Li}$ mixture), as might be attained experimentally by exploiting optically or magnetically induced Feschbach resonances [20]. For these calculations we have chosen the geometrical parameters of the traps and the numbers of atoms as in the Paris experiment on ${ }^{6} \mathrm{Li}^{7} \mathrm{Li}$ [5]: namely $N_{b} \simeq N_{f}=10^{4}, \omega_{b} / 2 \pi=4000 \mathrm{~Hz}, \omega_{f} / 2 \pi=3520 \mathrm{~Hz}$ and $\lambda_{b} \simeq \lambda_{f} \simeq 1 / 60$. The results are summarized in a quantum phase diagram at $T=0$ in the plane $\left\{a_{b f} / a_{b b}, k_{f} a_{b b}\right\}$, which is reported in Fig. 2. This contains all the lowest-energy configurations that we have found, with the inset showing an enlargement of the region near the origin of the above-mentioned plane. The curve drawn inside the inset in Fig. 2 is obtained from Eq. (10) and is in full agreement with the numerical results in separating the region of full demixing from the mixing or partial-demixing regions.

We turn to a detailed account of the configurations corresponding to the various symbols and letters in the phase diagram in Fig. 2. Below the phase separation line in the inset two different types of configurations are found, one with a core of bosons in partial overlap with an envelope of mainly fermions (triangles down) and a specular one with a core of fermions enveloped by mainly bosons (triangle up). An interesting configuration that we have observed in the latter case shows coexistence of a mixed state with a purely bosonic phase (see Fig. 3): this combination is not stable in the homogeneous mixture [10].

In the regime of phase separation a variety of different configurations are observed, including several that require a break of the symmetry imposed by the confining harmonic potentials. The letters a-d in the phase diagram in Fig. 2 give the locations of four such energetically stable structures, which are shown in Fig. 4 together with some other metastable structures. The symmetric configuration with boson inside and fermion outside, indicated by (a), is obtained in Fig. A with the choice $a_{b b}=600$ Bohr radii and $a_{b f}=10 a_{b b}$, while the complementary configuration (b) formed from fermions inside and bosons outside is obtained with $a_{b b}=64600$ Bohr radii and $a_{b f}=a_{b b} / 2$. Other configurations obtained with the same scattering lengths as the symmetric structure of type (a) are shown in Fig. 4: these are (c) a boson torus inside a fermion cloud, (d) a threefold symmetric structure formed from fermions surrounded by a shell of bosons inside a fermion envelope, (e) an asymmetric structure in which the bosons are shifted away from the center of the trap along the $z$ axis, and (f) a "sandwich" formed by bosons inside a fermion cloud. Finally the structure indicated by (g) in Fig. 4 , which consists of a torus of fermions around an elongated boson core, is found with the choice $a_{b b}=6250$ Bohr radii and $a_{b f}=2 a_{b b}$.

With the exception of (a) and (b) the configurations shown in Fig. 4 are very different from the density distributions that are met in the mixed phase, and thus could be identified in an experiment by looking at the column-density pictures of the atomic clouds. As an illustration we give in Fig. 5 the column-density images corresponding to the case (c) of a bosonic torus, and in Fig. 6 those for the case (f) of a boson sandwich.

Finally, we have examined the role of the anisotropy of the confinement on the configurations of lowest energy in the phase-separated regime. To this end we have evaluated the stability of the lowest-energy configuration by varying the anisotropy from $\lambda_{f}=1 / 60$ to $\lambda_{f}=1$ while keeping the ratio $\lambda_{b} / \lambda_{f} \simeq 1$, with the choice $a_{b b}=600$ Bohr radii and $a_{b f}=10 a_{b b}$ for the scattering lengths. The most stable configuration remains the symmetric structure (a) except that for $\lambda_{f} \simeq 1$ the structure (d) has the lowest energy. For this particular choice of parameters the relative difference in energy between the metastable configurations does not vary much with $\lambda_{f}$. 


\section{THE ROLE OF TEMPERATURE}

At finite temperature an increasing fraction of the bosons populates the thermal cloud, thus depleting the condensate. In harmonic confinement the thermal cloud is wider and more dilute than the condensate, and thus is much less interacting with the fermions. As a result the thermal cloud is not yet in phase separation when the condensate already is.

At low temperatures the non-condensate fraction is small and its presence does not affect the description of the phase separation between the condensate and the fermions that we have given in the preceding section. In particular we have verified that at $T$ of the order of $0.2 T_{f}$, as are reached in current experiments, all the exotic configurations (c) to (g) remain possible. They are not found at higher temperatures: we show as an example in Fig. 7 the behavior of the boson torus configuration (c) as a function of temperature. For $0.2 T_{f}<T \leq 0.3 T_{f}$ a part of the condensed bosons moves from the torus to the center of the trap and for $T>0.3 T_{f}$ the phase separation is lost.

At still higher temperatures the thermal cloud starts to be consistently populated and we have found that phase separation between the fermions and the entire bosonic cloud becomes possible for very large values of $a_{b f} / a_{b b}$ [14]. In this case, as is illustrated in Fig. 8, the fermion cloud exhibits a central hole having the size of the bosonic thermal cloud.

\section{SUMMARY AND CONCLUDING REMARKS}

We have performed an extensive analysis of the phenomenon of phase separation for a boson-fermion mixture under confinement on the basis of the atomic density profiles of the two components. First of all we have pointed out that three regimes can be identified owing to the finite size of the system: a mixed phase where the bosonic and fermionic clouds overlap, a partially demixed state where the overlap is decreasing, and a phase-separated regime where there is no overlap between the two components at zero temperature. We have given analytical expressions for the positions of the two cross-overs between these regimes in terms of the physical parameters of the system, and verified these conditions by an extensive numerical study based on varying the coupling constants, the numbers of atoms, and the geometry of the trap. General expressions for the transition lines are particularly useful in view of the several experiments in progress [6], which are run with different experimental parameters such as the relative numbers of bosons and fermions, the atomic masses, and the trap anisotropies.

The transition to the partially phase-separated regime, that we have located where the boson-fermion interaction energy starts to decrease on increasing the boson-fermion scattering, is important for the experiments on fermion cooling: the boson-fermion collision rate is decreased by a geometrical factor which is proportional to the overlap between the two clouds. At fixed boson-boson scattering length we have given a simple model for the transition in terms of a combination of two scaling parameters, which characterize the cases $N_{b} \ll N_{f}$ and $N_{f} \ll N_{b}$.

The transition to full demixing has been illustrated by a universal phase diagram expressed in terms of scaling parameters of the system. It is remarkable that in our model the location of this transition does not depend on the number of bosons. In the region of phase separation we have found several configurations with various topology and identified the minimum-energy configurations. We have also investigated the effect of increasing temperature on phase separation. Some exotic configurations that are found in the phase diagram at $T=0$ turn into others of higher symmetry before the condensate-fermion demixing is lost.

The main limitations of the present model come from the two basic approximations that we have employed: the Thomas-Fermi approximation for the condensate and the mean-field Hartree-Fock approximation for the other gaseous clouds. The Thomas-Fermi approximation breaks down for a very small condensate or for negative values of the bosonboson scattering length, and one would need to solve the full Gross-Pitaevskii equation. A description beyond mean field is required when the dilution parameters increase and for a trapped gas this is still an open problem. The analogue of the Beliaev expansion at $T=0$ has recently been derived for the homogeneous boson-fermion mixture [21].

Of course, the evolution of the mixture towards phase separation modifies not only its equilibrium properties, but also its dynamical properties and collective excitation spectrum. The approach to the transition will be signalled by a softening of the frequencies of surface or bulk modes having the appropriate symmetry for a phase-separated configuration to be attained. Experiments measuring dynamical properties could therefore provide an alternative method for revealing the onset of demixing. Calculations on these spectra are in progress. 


\section{ACKNOWLEDGMENTS}

This work was supported by INFM through the PRA2001-Photonmatter.

[1] M. H. Anderson, J. R. Ensher, M. R. Matthews, C. E. Wieman, and E. A. Cornell, Science 269, 198 (1995); K. B. Davis, M.-O. Mewes, M. R. Andrews, N. J. van Druten, D. S. Durfee, D. M. Kurn, and W. Ketterle, Phys. Rev. Lett. 75, 3969 (1995); C. C. Bradley, C. A. Sackett, J. J. Tollett, and R. Hulet ibid. 75, 1687 (1995).

[2] H. T. C. Stoof, M. Houbiers, C. A. Sackett, and R. G. Hulet, Phys. Rev. Lett. 76, 10 (1996).

[3] B. DeMarco, S. B. Papp, and D. S. Jin, Phys. Rev. Lett. 86, 5409 (2001).

[4] A. G. Truscott, K. E. Strecker, W. I. McAlexander, G. B. Partridge, and R. G. Hulet, Science 291, 2570 (2001).

[5] F. Schreck, L. Khaykovich, K. L. Corwin, G. Ferrari, T. Bourdel, J. Cubizolles, and C. Salomon, Phys. Rev. Lett. 87, 080403 (2001).

[6] Z. Hadzibabic, C. A. Stan, K. Dieckmann, S. Gupta, M. W. Zwierlein, A. Görlitz, and W. Ketterle, cond-mat/0112425.

[7] J. Goldwin, S. B. Papp, B. DeMarco, and D. S. Jin, Phys. Rev. A 65, 021402(R) (2002).

[8] K. Mølmer, Phys. Rev. Lett. 80, 1804 (1998).

[9] S. L. Sondhi, S. M. Girvin, J. P. Carini, and D. Shahar, Rev. Mod. Phys. 69, 315 (1997).

[10] L. Viverit, C. J. Pethick, and H. Smith, Phys. Rev. A 61, 053605 (2000).

[11] N. Nygaard and K. Mølmer, Phys. Rev. A 59, 2974 (1999).

[12] A. Minguzzi and M. P. Tosi, Phys. Lett. A 268, 142 (2000).

[13] T. Miyakawa, K. Oda, T. Suzuki, and H. Yabu, J. Phys. Soc. Japan 69, 2779 (2000).

[14] Z. Akdeniz, P. Vignolo, A. Minguzzi, and M. P. Tosi, J. Phys. B 35, L105 (2002).

[15] A. Minguzzi, S. Conti, and M. P. Tosi, J. Phys.: Cond. Matt. 9, L33 (1997).

[16] M. Amoruso, A. Minguzzi, S. Stringari, M. P. Tosi, and L. Vichi, Eur. Phys. J. D 4, 261 (1998).

[17] G. Baym and C. J. Pethick, Phys. Rev. Lett. 76, 6 (1996).

[18] B. DeMarco, J. L. Bohn Jr., J. P. Burke, M. Holland, and D. S. Jin, Phys. Rev. Lett. 82, 4208 (1999).

[19] L. Vichi, M. Amoruso, A. Minguzzi, S. Stringari, and M. P. Tosi, Eur. Phys. J. D 11, 335 (2000).

[20] S. Inouye, M. R. Andrews, J. Stenger, H. -J. Miesner, D. M. Stamper-Kurn, and W. Ketterle, Nature 392, 151 (1998); S. L. Cornish, N. R. Claussen, J. L. Roberts, E. A. Cornell, and C. E. Wieman, Phys. Rev. Lett. 85, 1795 (2000).

[21] A. P. Albus, S. A. Gardiner, F. Illuminati, and M. Wilkens, cond-mat/0201102 


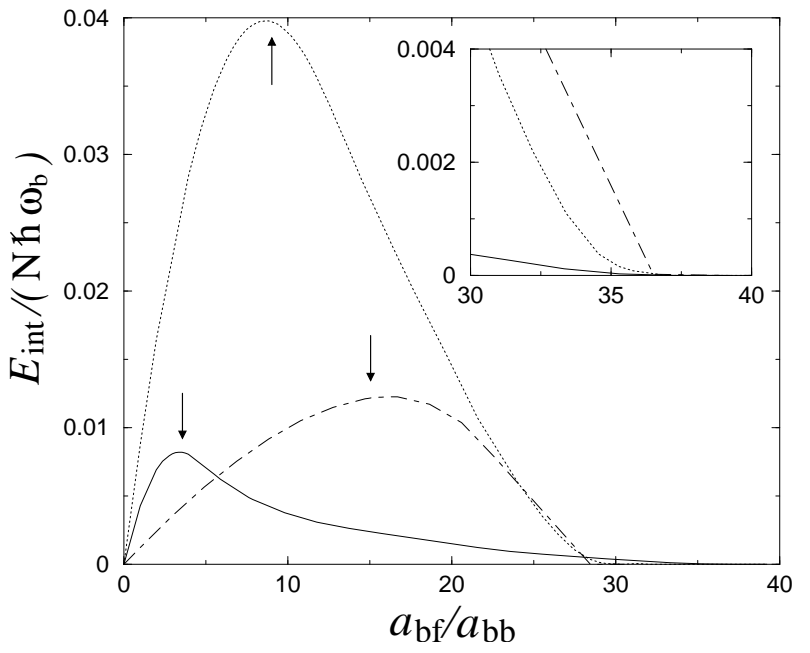

(a)

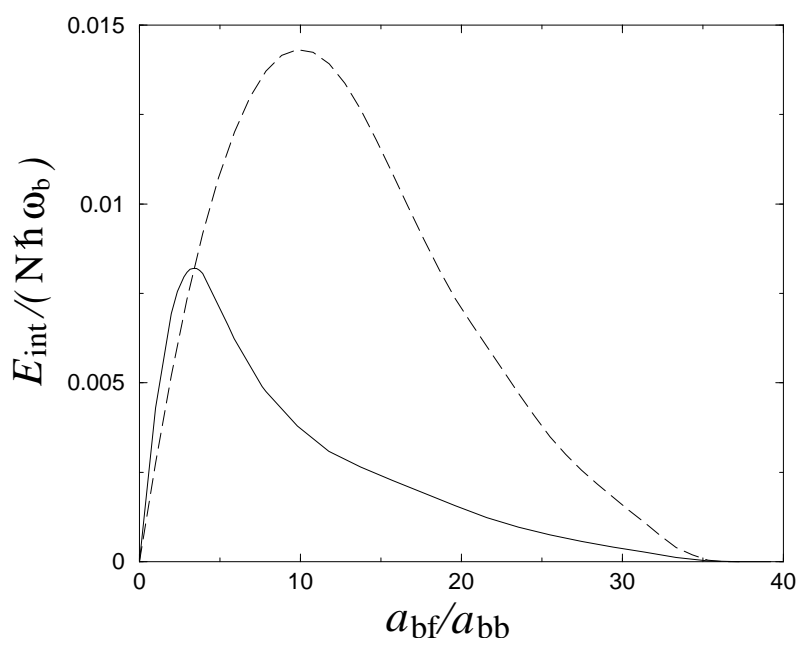

(b)

FIG. 1. Boson-fermion interaction energy at $T=0$ (in units of $N \hbar \omega_{b}$ where $N=N_{f}+N_{b}$ ) as a function of the adimensional ratio $a_{b f} / a_{b b}$ for $a_{b b}=5.1 a_{0}$ with $a_{0}$ being the Bohr radius. The other parameters are $\omega_{b} / 2 \pi=4000 \mathrm{~Hz}, \omega_{f} / 2 \pi=3520 \mathrm{~Hz}$, $\lambda_{b} \simeq \lambda_{f} \simeq 1 / 60$, and atomic masses corresponding to the ${ }^{6} \mathrm{Li}-{ }^{7} \mathrm{Li}$ mixture. (a) For $N=2.1 \cdot 10^{5}$, with $N_{f, 1}=10^{4}$ (continuous line), $N_{f, 2}=1.05 \cdot 10^{5}$ (dotted line) and $N_{f, 3}=2 \cdot 10^{5}$ (dotted-dashed line). The arrows indicate the positions of the maxima according to Eq. (7). In the inset are shown the same curves in the same vertical units, after rescaling the second and third curve along the horizontal axis by factors $\left(N_{f, 2} / N_{f, 1}\right)^{1 / 12}$ and $\left(N_{f, 3} / N_{f, 1}\right)^{1 / 12}$. (b) For fixed number of fermions $N_{f}=10^{4}$ and different numbers of bosons $N_{b}=2 \cdot 10^{5}$ (continuous line) and $N_{b}=10^{4}$ (dashed line).

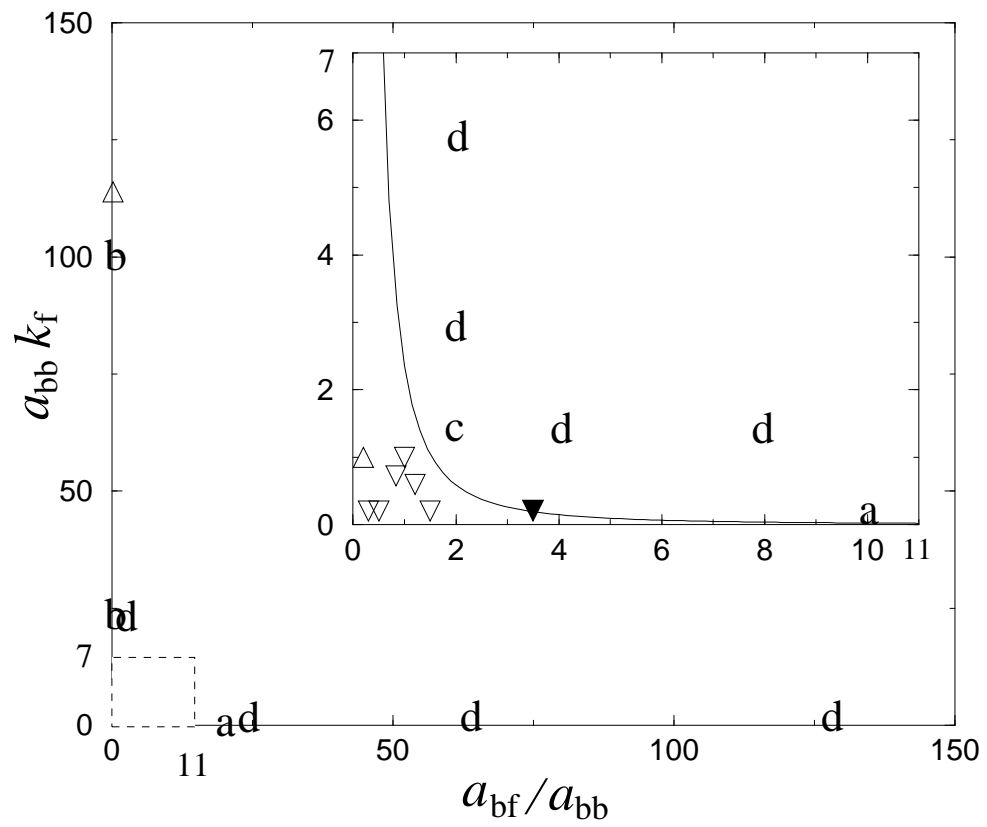

FIG. 2. Phase diagram at $T=0$, in the plane defined by the adimensional parameters $a_{b b} k_{f}$ and $a_{b f} / a_{b b}$. The bottom left corner of the figure is shown enlarged in the inset. The continuous line in the inset corresponds to the condition of phase separation given by Eq. (10). In the mixed-phase regime we have the following configurations: $\Delta$ fermions at the center: $\nabla$ bosons at the center; $\boldsymbol{\nabla}$ bosons an the center in an almost demixed configuration. For the phase-separated regime we have used the following notations (see Fig. 4): (a) symmetric configuration with fermions outside; (b) symmetric configuration with bosons outside; (c) boson torus inside a fermion cloud; (d) three-component symmetric configuration with fermions outside and in the central core. All the configurations in this phase diagram have been evaluated at zero temperature, using $N_{b}=N_{f}=10^{4}$, $\omega_{b} / 2 \pi=4000 \mathrm{~Hz}, \omega_{f} / 2 \pi=3520 \mathrm{~Hz}, \lambda_{b} \simeq \lambda_{f} \simeq 1 / 60$, and atomic masses corresponding to the ${ }^{6} \mathrm{Li}^{7}{ }^{7} \mathrm{Li}$ mixture. 


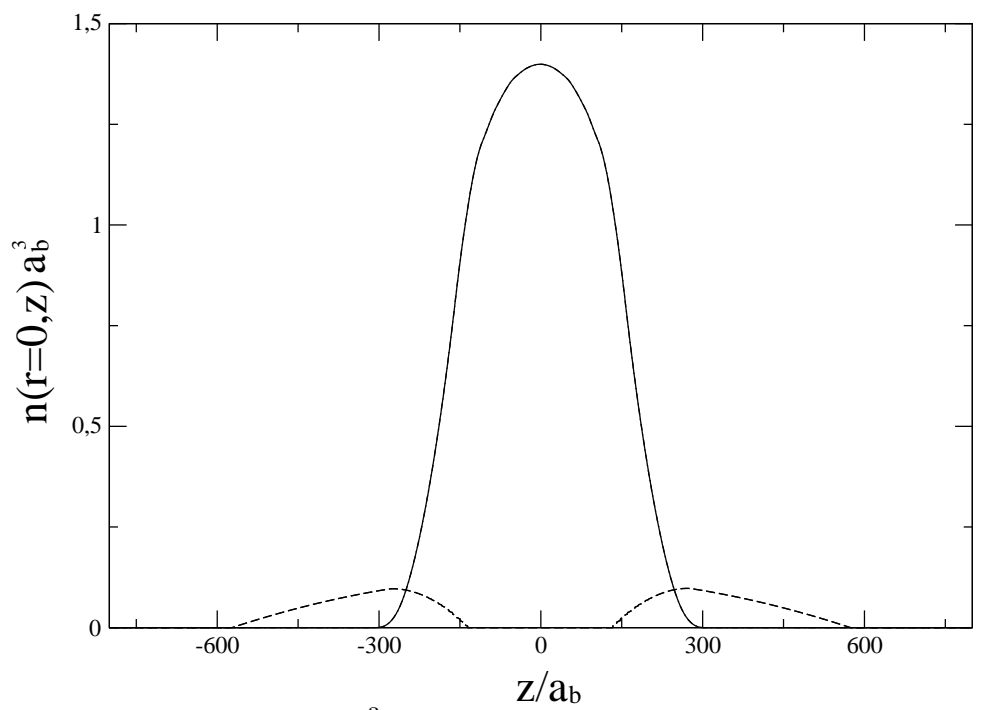

FIG. 3. Section of the density profiles (in units of $a_{b}^{-3}$ ) as a function of $z / a_{b}$ for fermions (solid line) and bosons (dashed line) in a configuration where a mixed phase and a purely bosonic one coexist ( $\triangle$ in Fig. 2). This configuration has been obtained at $T=0$ with $a_{b b}=323000 a_{0}$ and $a_{b f}=32300 a_{0}$. The numbers of atoms and all other parameters are as in Fig. 22.

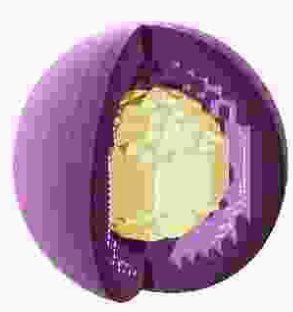

(a)

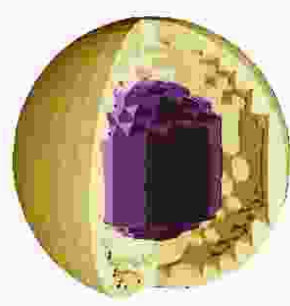

(b)

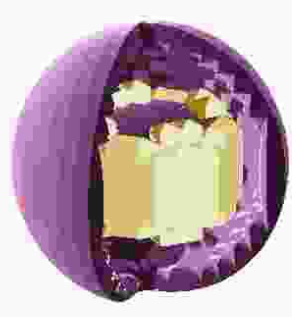

(c)

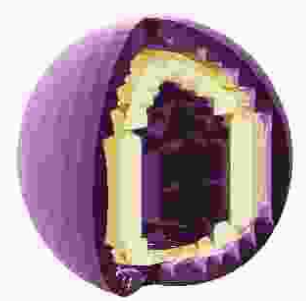

(d)

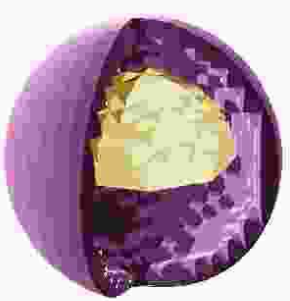

(e)

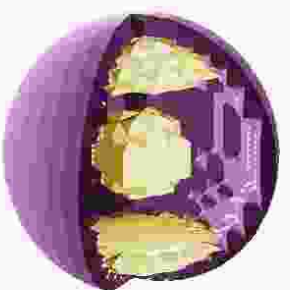

(f)

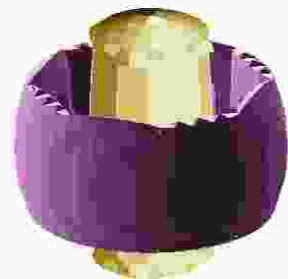

(g)

FIG. 4. Configurations in the phase-separated regime for $N_{f}=N_{b}=10^{4}$ particles at $T=0$. (a) symmetric configuration with fermions outside; (b) symmetric configuration with bosons outside; (c) boson torus inside fermion cloud; (d) threefold symmetric configuration; (e) asymmetric configuration; (f) boson sandwich inside a fermion cloud; and (g) torus of fermions around an elongated core of bosons. The structures (a) and (c-f) are for the same values of $a_{b b}=600 a_{0}$ and $a_{b f}=6000 a_{0}$, and their energies per particle are $4.24,4.71,5.38,5.50$, and $5.85 \hbar \omega_{b}$. The configuration (b) was obtained for $a_{b b}=64600 a_{0}$ and $a_{b f}=32300 a_{0}$ and the value of its energy per particle is $13.25 \hbar \omega_{b}$. The configuration (g) was obtained for $a_{b b}=6250 a_{0}$ and $a_{b f}=12500 a_{0}$ and the value of its energy per particle is $9.27 \hbar \omega_{b}$. All other parameters are as in Fig. 2. 

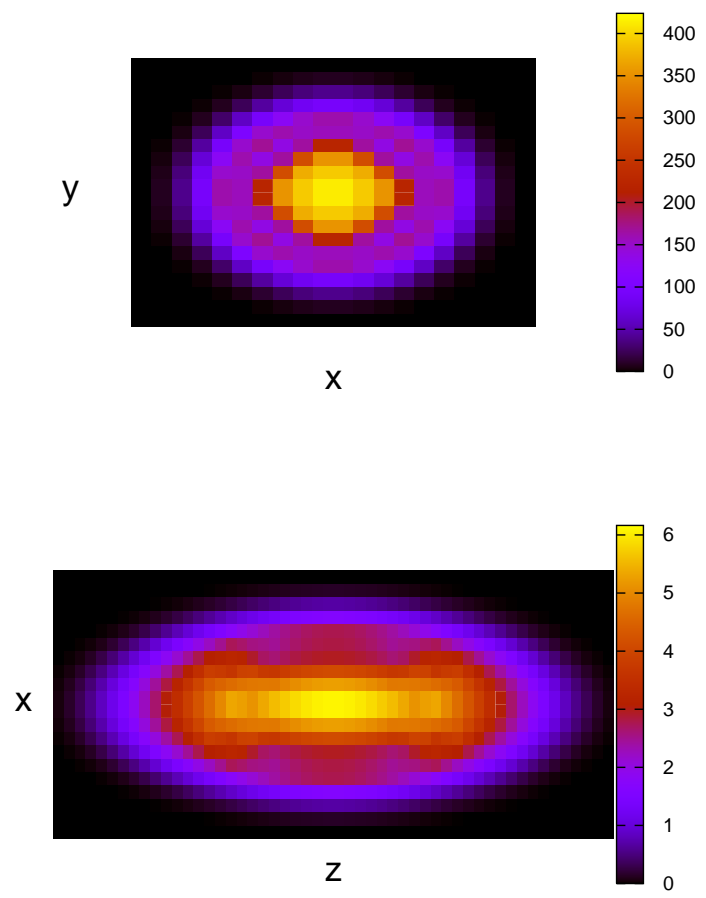
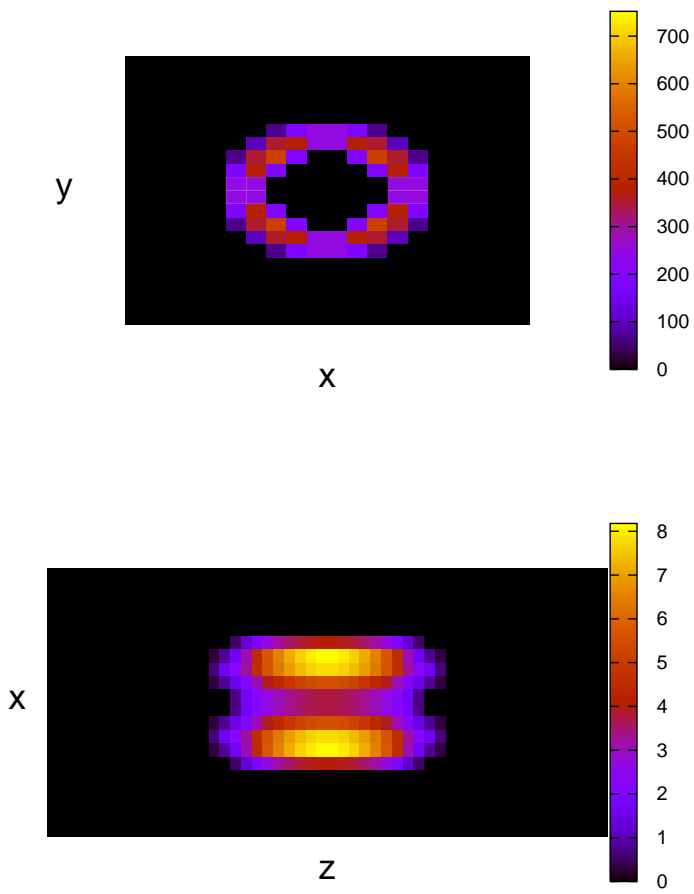

FIG. 5. Column densities (in units of $a_{b}^{-2}$ ) in the radial plane (top) and in the $\{x, z\}$ plane (bottom) for the fermionic cloud (left) and the bosonic cloud (right) of the bosonic torus (configuration (c) in Fig. 1 , obtained with $a_{b b}=600 a_{0}$ and $\left.a_{b f}=6000 a_{0}\right)$. The size of the figures in the $\{x, y\}$ plane and in the $\{x, z\}$ plane is $11 \mu m \times 11 \mu m$ and $11 \mu m \times 400 \mu m$, respectively. The other parameters are as in Fig. 2.
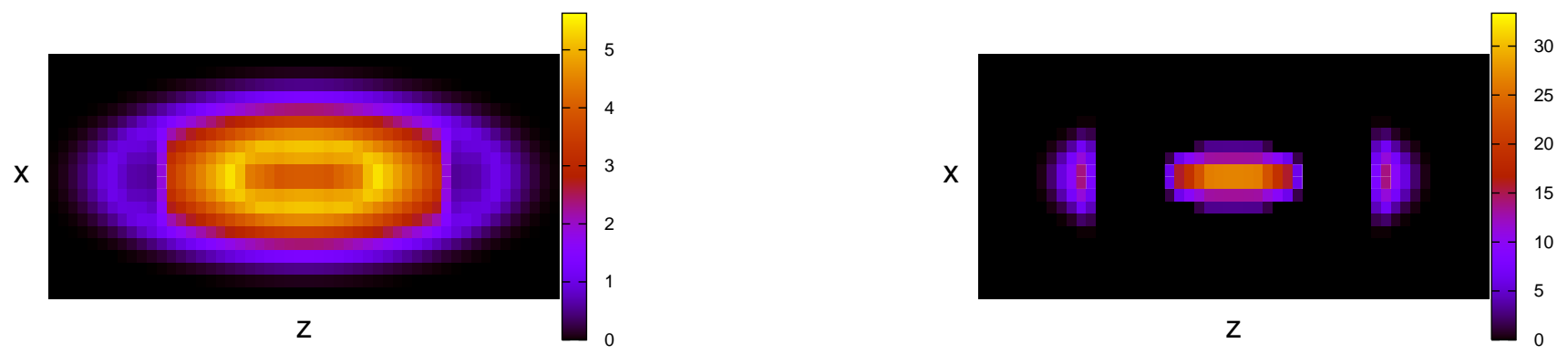

FIG. 6. Column densities in the $\{x, z\}$ plane (in units of $a_{b}^{-2}$ ) for the fermions (left) and for the condensate (right) in the sandwich configuration (f) in Fig. 1 , obtained with $a_{b b}=600 a_{0}$ and $a_{b f}=6000 a_{0}$. The size of the figures is $11 \mu m \times 400 \mu m$. The other parameters are as in Fig. 2 . 

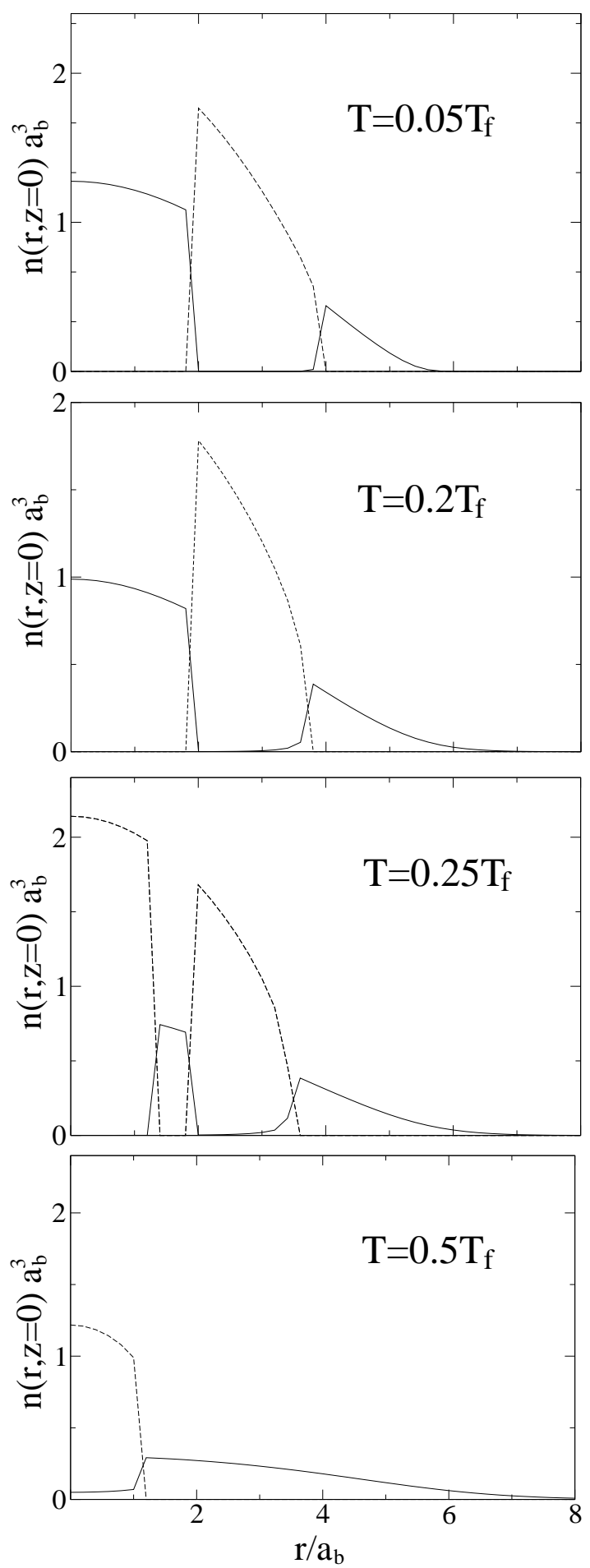

FIG. 7. Sections of the density profiles at different temperatures (in units of $a_{b}^{-3}$ ) for the fermions (solid line) and for the condensate (dashed line) as functions of the radial coordinate $r / a_{b}$ for the configuration (c) in Fig. 4, obtained with $a_{b b}=600 a_{0}$ and $a_{b f}=6000 a_{0}$. The values of the other parameters are as in Fig. 2. 


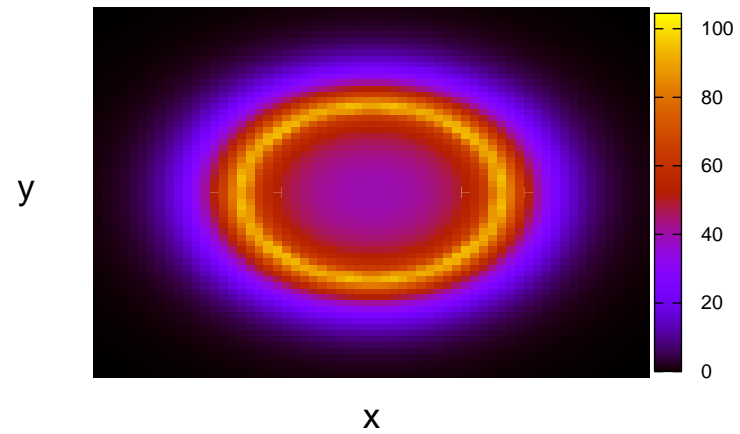

$x$

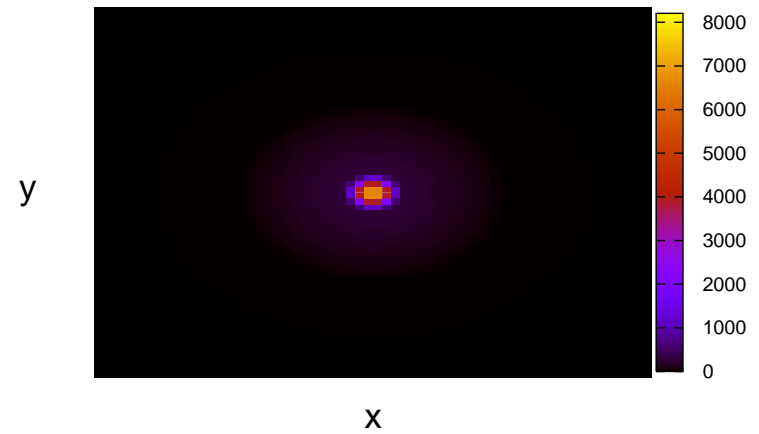

X

FIG. 8. Column densities in the $\{x, y\}$ plane (in units of $a_{b}^{-2}$ ) for fermions (left) and bosons (right) in the fully demixed regime at $T=0.6 T_{f}$, for $a_{b b}=5.1 a_{0}$ and $a_{b f}=2 \cdot 10^{5} a_{0}$. The values of the other parameters are as in Fig. 22. In the right panel the thermal bosons are not visible because of the high density of the central condensate. The size of the figures is $11 \mu m \times 11 \mu m$. 